\title{
TWO CASES OF MELIOIDOSIS.
}

\author{
$\mathrm{BY}$ \\ A. T. STANTON, M.D., M.R.C.P., D.P.H. \\ (Director of Government Laboratories, Federated Malay States); \\ WILLIAM FLETCHER, M.D. \\ (Bacteriologist, Institute for Medical Research, F.M.S.); \\ AND \\ K. KANAGARAYER, L.M.S. \\ (Assistant Surgeon, District Hospital, Kuala Lumpur, F.M.S.)
}

(With Plates III-V, containing Figs. 1-6.)

$W_{E}$ have described under the name Melioidosis a fatal disease affecting man and rodents, the latter constituting in all probability a natural reservoir of the virus (Stanton and Fletcher, 1921). Whitmore (1913) first drew attention to this malady as "a glanders-like disease occurring in Rangoon" and isolated the organism, now known by his name, which is the cause of the disease. It is a small Gram-negative bacillus which sometimes bears a strong resemblance to the Bacillus pestis, particularly in early subcultures and in films prepared direct from lesions in the body where as a rule it is very scanty. The lesions themselves, as emphasised by Whitmore, are very like those of glanders; but $B$. whitmori possesses two attributes which distinguish it at once both from plague and from glanders bacilli: it is actively motile and it grows on glycerine agar in a most striking and characteristic manner, forming curious wrinkled, corrugated colonies. There is also a mucoid form, analogous with the mucoid variety of B. paratyphosus B. Both forms are shown in Fig. 1 .

The majority of the cases which have been studied hitherto have not been examined by a medical man until after death, and we are reporting these two because, in the first place, each of them had been in hospital for several days before death took place and, secondly, because one of them is remarkable in being the only instance of the disease which has been recognised in a European.

\section{Case 1.}

The FIRSt PATIENT was a robust Tamil cooly, forty years of age, who was admitted to the District Hospital at Kuala Lumpur on November 7, 1923, complaining of fever, which had commenced twelve days previously, and pains in the epigastrium. During the time he was in hospital, his temperature was irregular and ranged from just above normal to $102^{\circ} \mathrm{F}$. No malaria 
parasites were found in his blood and no physical signs of disease could be detected, except a tender and greatly enlarged spleen. Five days after his admission, the bases of both lungs were consolidated and, whereas he was constipated when he came into the hospital, he now began to have diarrhoea. He became restless with hurried breathing, and collapsed on the night of November 14. He died on the following morning, about three weeks after the beginning of his illness.

A POST-MORTEM EXAMination was made within a few hours of death and lesions were found in the lungs, liver, spleen and large intestine. There were several areas of consolidation in both Lungs, two in the left and ten in the right, situated chiefly at the periphery and free edges of the lobes (see Fig. 2). The areas of consolidation were from 1 to $2 \mathrm{~cm}$. in diameter, but in some places they had coalesced to form larger masses. Projecting beneath the pleura, they gave a knobbed appearance to the surface of the lungs. On cutting through one of the larger patches, it was seen that there ran through the centre of it a blood vessel bearing upon its branches, like fruit upon the branches of a tree, minute tubercle-like nodules which had coalesced to form a larger area of consolidation. There was no inflammation of the pleura overlying these areas, which were composed of aggregations of small suppurating points, the tubercle-like nodules just mentioned. In films made from the lungs there were many minute, slender Gram-negative bacilli, most of them extra-cellular, and also a few Gram-positive cocci. No organisms were seen which bore any strong morphological resemblance to $B$. whitmori, but this bacillus grew in cultures made from the patches of consolidation.

The LIVER was enlarged and weighed 1900 grms. (the average weight, in Tamils, is 1372 grms.). There were five rounded, projecting bosses on its surface; the two largest measured about $4 \mathrm{~cm}$. in diameter and contained yellow pus which could be seen through Glisson's capsule. When the organ was incised the smaller lesions were found to consist of aggregations of minute suppurating tubercles which, in the two larger ones, had coalesced to form sharply circumscribed abscesses of a honeycomb character. The lesions were in the periphery of the organ and were apparently the result of septic infarction. The GALL-BLADDER was thickened and the mucous lining inflamed (see Fig. 3). Films prepared from the pus in the liver contained some Grampositive diplococci, like pneumococci, and also a few Gram-negative bacilli which stained in a bipolar fashion with Leishman's stain. Bacillus whitmori was cultivated from the liver pus and from the gall-bladder.

The Spleen (see Fig. 4) weighed 1450 grms., or nearly three times its normal weight. In it, there were numerous septic infarcts some of which were suppurating, and one measured $4 \times 1.5 \mathrm{~cm}$. in diameter. The capsule was greatly thickened. A few very small, slender, Gram-negative bacilli, and some Gram-positive diplococci were seen in films prepared from the spleen. B. whitmori was cultivated from the pus in the abscesses.

The gastro-intestinal canal was apparently healthy, except the CAECUM, 
in which there were several small erosions, each covered with a granular layer of adherent debris (see Fig. 5). In one spot there was a definite ulcer, about $1 \mathrm{~cm}$. long, with overhanging edges. Cultures were made from this ulcer and also from the erosions, but B. whitmori was not isolated.

Further investigations were made into the characters of the strains of B. whitmori cultivated from the lungs, spleen, liver and gall-bladder. These cultures resembled the laboratory type strain ("Ragaviah") of the organism in growth and morphology, in their action on sugar, in their liquefaction of gelatine and in all other respects. They were agglutinated to full titre by an immune serum prepared with the type strain of $B$. whitmori but not by a B. mallei serum prepared with the Lister Institute strain Minett.

Several animals WERE inoculaten, some with pus from the abscesses and others with cultures. Two guInea-PIGs, $A$ and $B$, were inoculated with pus from the liver. A little of the pus was introduced into the right nostril of guinea-pig $A$, and six days later, the dirty condition of the nose showed that there was some discharge. On the eighth day the right eye was inflamed and, on the following morning, the animal died. Post-mortem, the subcutaneous tissue was red and injected, and there was an enlarged caseous gland in the right inguinal region. The nose was red and swollen, and inside it there was a little thick pus. B. whitmori was recovered in almost pure culture from the nose and from the gland.

Guinea-pig $B$ was inoculated under the skin of the abdomen with the same pus, and at the same time, as guinea-pig $A$. A small hard, red swelling resulted, and a little ulcer appeared at the point of inoculation. On the ninth day, by which time guinea-pig $A$ was dead, the ulcer had healed and the swelling had almost disappeared. The animal was lively and was eating well; but there was an enlarged gland in the right groin. This gland suppurated and other buboes appeared on the shoulders and in the axillae. By the end of the third week the guinea-pig was not eating well and it died on the twentyfifth day after inoculation. At the autopsy, abscesses were found in the groins and axillae of both sides, and also a large one, full of pus which resembled curdled milk, in the region of Douglas's pouch. B. whitmori was recovered in pure culture from these abscesses. A third guinea-pig was inoculated subcutaneously with a culture from the animal and died within sixteen hours from septicaemia due to $B$. whitmori. Post-mortem, there were enlarged haemorrhagic inguinal glands, a slightly enlarged spleen, and subcutaneous haemorrhages, such as are seen in a guinea-pig that has died from plague. B. whitmori was cultivated from the heart and spleen.

Another guinea-pig, $D$, was inoculated subcutaneously with the same strain, which had been grown for forty-eight hours in 10 c.c. of broth to which $0 \cdot 25$ c.c. of a high titre immune serum had been added. A small abscess developed at the point of inoculation, which ruptured and discharged its contents. The guinea-pig continued active and ate its food; but the axillary, subscapular and inguinal glands became enlarged andit died on the twenty-fifth 
day, with suppurating buboes containing thick creamy pus, and with small abscesses in the liver. The spleen was enlarged, the left lung was hepatised and the right lung and pleura were filled with suppurating tubercles. B. whitmori was cultivated from the buboes and from the viscera. Several other guineapigs were inoculated with results similar to those we have already described.

In order to determine if HORSES are susceptible to melioidosis, which resembles glanders in so many ways, an Australian mare was inoculated subcutaneously by Major Symonds, Veterinary Surgeon F.M.S., with about five hundred million organisms of the fourth subculture on agar. The mare's health did not appear to be disturbed, nor was there any rise of temperature or general reaction. A small fluctuating swelling, half the size of a walnut, appeared at the point of inoculation, but it subsided in a week, and when the mare was killed, four months later, there were no signs of melioidosis, nor could Whitmore's bacillus be cultivated from the blood or viscera.

A Java pony, inoculated in the same way, by Major Symonds, at the same time as the mare, developed a tender swelling and a slight rise of temperature. There was a little fever for ten days and the swelling suppurated; but, by the end of three weeks, the resulting ulcer had healed and the pony seemed none the worse. She was killed four months later and no signs of infection could be discovered

Case 2.

The second patient was a European, forty-two years of age, who was admitted to hospital on December 17, 1923, with diarrhoea and malaise which had lasted for ten days. He complained of pain in the epigastrium which came on almost immediately after food, and on five occasions he had vomited. He had been in the Malay States for sixteen years, and for three months prior to his illness he had been living in a mining village, in a house said to be infested with rats. We wish to draw attention to this, because melioidosis is a disease which occurs naturally in rodents.

We are indebted to Dr J. G. Castellain, the Medical Officer in charge of the European Hospital at Kuala Lumpur, for the clinical notes. The patient's temperature was $99 \cdot 4^{\circ}$ on admission, and it swung between subnormal and $102^{\circ}$ for the first five days, after which it never sank below a hundred degrees. It rose to $106 \cdot 4^{\circ}$ just before his death, which took place fourteen days after his admission and twenty-four after he began to feel ill. While he was in hospital, he complained of pain in the pit of the stomach, where there was considerable tenderness, and the liver was definitely enlarged. He vomited on several occasions and, with the swinging of his temperature, he had rigors and sweats. During the first few days in hospital he was passing six or eight loose motions daily but this diarrhoea improved and, for the last week, his bowels acted not more often than two or three times in twenty-four hours. His stools contained no blood or mucus, nor were amoebae or dysentery bacilli found on examination. No malaria parasites were seen in his blood; 
the proportion of polynuclear leucocytes was not increased. The patient became progressively weaker, sank into a low muttering delirium and died.

A limited POST-MORTEM EXAMINATION was made within a few hours of death. The Lungs and spleEN appeared to be healthy on superficial examination, but they were not incised. The peritoneum was apparently normal except for some adhesions of the omentum to the surface of the liver.

On the upper surface of the left lobe of the LIVER there was a dark congested swelling with yellow points of pus showing through Glisson's capsule. There was a similar, though smaller, swelling in the middle of the upper surface of the right lobe. Each of these tumours was a large abscess. That on the left side was spherical and about $6 \mathrm{~cm}$. in diameter. It contained only a few ounces of thick yellow pus. The wall consisted of a layer of shaggy necrotic material about $2 \mathrm{~cm}$. in thickness. The abscess in the right lobe was a little smaller, but two finger-like projections extended from it. One towards the quadrate lobe and the other upwards. There was very little pus in this second abscess. Films prepared from scrapings of the abscess wall consisted of necrotic cells, most of which contained fat globules. There were also a few red blood corpuscles, but no healthy polynuclear pus cells. No amoebae could be found although a search for them was made in several films. No microorganisms were seen in stained films, but $B$. whitmori grew on glycerine agar inoculated with the material.

The GALL-BLADDER was at least an eighth of an inch thick and resembled the gall-bladder of the other patient, Velu. Unfortunately no cultures were made from it.

In the large intestine there were no amoebic lesions, but there were three or four small ulcers, in the CAECUM, which bore some resemblance to those found in a similar position at the post-mortem examination of Velu.

The bacteriological investigation of this case was incomplete. At the autopsy we jumped to the hasty conclusion that the abscesses were associated with $E$. histolytica, and nothing but a portion of the wall of the abscess in the right lobe of the liver (see Fig. 6) was taken to the laboratory for further investigation. Glycerine agar slopes were inoculated with material from this and, on these, B. whitmori grew in almost pure culture. The colonies assumed their usual form within forty-eight hours, a raised dome in the centre with a wide flat margin around it, like the broad-brimmed straw hats worn by Chinese in ceremonial processions. After two or three days the growth became characteristically corrugated. Individually, the bacilli were actively motile and, in the earlier subcultures, they showed excellent bipolar staining with Leishman's stain. The organisms grew rapidly in peptone water, causing great turbjdity and forming a wrinkled pellicle on the surface of the medium. Acid, but not gas, was produced in glucose and saccharose, within twenty-four hours. Lactose was fermented on the fourth day and also mannite and dulcite at the surface of the medium, where they were in contact with the air. In later subcultures the power of fermenting sugars was considerably less. 
Gelatine was liquefied on the fourth day. The organism was agglutinated to full titre by an immune serum prepared from the type laboratory strain (Ragaviah), and to half titre by an old sample of blood serum collected from the patient from whom this type strain was isolated.

Several ANIMALS WERE INOCULATED with the organism isolated from this case. Two wild Rats (Mus griseiventer, Bonhote) were inoculated subcutaneously with about a thousand million organisms, grown for twenty-four hours on an agar slope. In these animals the disease, following inoculation or feeding with contaminated food, usually runs a chronic course lasting about three months, but both these rats died from acute septicaemia in less than twenty-four hours, with great subcutaneous redness and congestion of all the organs. B. whitmori was cultivated from the heart's blood of both the rats, although-as is usually the case in infection with this bacillus-none could be found in films made directly from the blood.

Two GUINEA-PIGS were inoculated at the same time as the rats; one subcutaneously with a thousand million organisms, the other by the introduction of a loopful of the culture into the left nostril. Both animals died from septicaemia. The first one died forty-eight hours after inoculation; with redness and oedema at the site of injection, redness of the subcutaneous tissue and enlarged haemorrhagic inguinal glands. The second animal died on the fifth day, with the usual signs of septicaemia and a cheesy deposit in the left nostril, which contained $B$. whitmori. This organism was cultivated from the heart in both cases. Five weeks later, a third guinea-pig was inoculated in the left nostril with the bacillus which had been subcultured repeatedly on glycerine agar, and in this case the disease ran a more chronic course. Slight discharge from the left side of the nose and from the left eye was noticed on the third day. The animal became thin, refused its food, and died on the twelfth day with enlarged caseous glands on each side of the trachea. B. whitmori was recovered from the glands and from the spleen.

A RABBIT, weighing 1300 grms., was inoculated under the skin of the abdomen with about a hundred million organisms. It died in thirty-six hours. Post-mortem, it was seen that the inflammatory process, set up by the injection of the virus, had extended inwards from the point of inoculation, through the abdominal wall to the peritoneum. There were minute tubercles on the parietal peritoneum, scattered along the veins and forming a definite cord along the lymphatics leading from the spot where the injection had been made. The under surface of the diaphragm was thickly covered with minute tubercles and the general appearance was very like that of miliary tuberculosis. B. whitmori was cultivated from the heart, spleen and local lesions.

Monkeys are susceptible to melioidosis and have been infected by feeding. Half a cubic centimetre of the second subculture of $B$. whitmori from this case, containing about five hundred million organisms, was squirted into the mouth of a Macacus cynomolgus with a pipette. It showed no signs of infection and is apparently in good health, two months later. 
A PONy, the same which had been inoculated with the strain of $B$. whitmori from the first patient, Velu, was given an intravenous injection of about a thousand million organisms of the culture obtained from the second, the European case, by Major Symonds. The temperature rose to $103 \cdot 6^{\circ}$ in three hours, and for six days it remained high, but by the end of a week the pony appeared to have recovered. It was killed sixteen weeks later and at the post-mortem examination no signs of melioidosis were discovered.

The first point to be noticed in a DIscussion of this report is that in neither case was the nature of the disease discovered during the life of the patient. Indeed, this has been done in only four out of the forty-eight cases in which melioidosis has been recognised. In one instance $B$. whitmori was cultivated from the blood, in another from the urine; in a third, from a pustular eruption, and in the fourth from an abscess. In this last case the suspicions, aroused by the "positive" result of an agglutination test, were confirmed by the cultivation of the bacillus from an abscess, which appeared later, connected with the lower end of the fibula.

As in animals, so in man, the patient may die from a fulminating, acute septicaemia, like the rats inoculated with the culture isolated from the second case in this report; or the disease may run a more chronic course, as in the guinea-pigs $B$ and $D$, which were infected with the pus from the first case. The signs and symptoms of the chronic form are dependent upon the distribution of the lesions. We have seen one patient in which the brunt of the disease fell upon the urinary system and, here, the prominent symptoms were due to lesions of the kidney, ureters and bladder. In the majority of the cases, which have come to our notice, the illness commenced as an acute septicaemia; if the patient did not succumb within the first few days, he passed into a typhoid condition of chronic septicaemia, with the formation of abscesses in different organs. Every case has died in less than four weeks except two; one of which recovered after a long illness, while the other is still an invalid with abscesses, sinuses and chronic suppuration in the bones of his feet and legs, more than two years after the commencement of the malady.

In the two patients which form the subject of this report, the onset of the disease was gradual, whereas, in the other cases which we have encountered, the illness has been characterised by a sudden onset accompanied by collapse and, sometimes, by a purging so violent as to suggest the diagnosis of cholera.

There are no definite clinical signs by which the disease can be recognised: a sure diagnosis can be made only by the cultivation of the causative organism. A "positive" agglutination reaction is of value as presumptive evidence particularly if the dilution increases after an interval (as it did in the instance which we have mentioned, where it rose from 1 in 80 to 1 in 1200 after an interval of three weeks): but this reaction is, obviously, of little value in the ordinary acute form of the disease. The organism has been cultivated from the blood and the urine of human cases and also from pustules and abscesses when these 
A. T. Stanton, W. Fletcher and K. Kanagarayer 275

have been present. In one instance it was cultivated from the faeces of a rabbit. Where the lungs are involved, as they usually are, the injection into a guinea-pig of sputum or of material obtained by puncture of the lung, might make the diagnosis clear. In cases of obscure fever associated with enlargement of the liver, this viscus should be explored with a needle, and glycerine agar slopes and animals should be inoculated with the material obtained.

Whenever an abscess of the liver is aspirated or drained, glycerine agar slopes and guinea-pigs should be inoculated with the pus. We consider it probable that some of the cases, in Malaya at least, which are diagnosed as amoebic abscesses, are really instances of melioidosis.

When we come to deal with the epidemiology of the disease, and attempt to find an answer to the question of the manner in which these two men become infected, we must confess ourselves still in difficulty. Melioidosis can be conveyed to rodents by subcutaneous inoculation, by introducing the virus into the nostrils or by feeding. Subcutaneous inoculation is followed, in animals, by a local lesion before generalisation takes place; but there was no history of any such manifestation in our two patients. Infection by inhalation is unlikely except where a number of cases are closely associated. We are of opinion that the disease was conveyed to these men by the ingestion of food which had been contaminated by rodents infected with melioidosis.

\section{Summary.}

Two cases of fatal melioidosis are described. The first a robust Indian labourer; the second a well-nourished, muscular European.

Case 1. Gradual onset. Irregular fever. Pain in the epigastrium. No physical signs of disease at first, except enlarged spleen; later, there were signs of consolidation at the bases of both lungs. Constipated at first; diarrhoea came on towards the end and the patient collapsed and died at the end of the third week.

At the autopsy; there were small patches of consolidation in the lungs; composed of minute suppurating tubercles. In the liver there were similar aggregations of small suppurating tubercles and two circumscribed abscesses. The gall-bladder was thickened. The spleen was enlarged and contained septic infarcts and abscesses. In the caecum there were small superficial ulcers. B. whitmori was cultivated from the lungs, spleen, liver and gall-bladder.

Animals were inoculated as follows: A guinea-pig, inoculated in the anterior nares with pus from the liver, died nine days later with caseous inflammation of the nasal passages and a caseous inguinal gland from which $B$. whitmori was recovered. A guinea-pig, inoculated subcutaneously with pus from the liver, died on the twenty-fifth day with abscesses in the lymphatic glands from which $B$. whitmori was cultivated. A guinea-pig, inoculated subcutaneously with a culture of the organism, died within sixteen hours from septicaemia. B. whitmori was recovered from the heart and spleen. A guinea-pig, inoculated subcutaneously with an attenuated culture, died on the twenty-fifth 
day with suppurating buboes and with abscesses in the liver and lungs from which $B$. whitmori was cultivated. Two horses were inoculated subcutaneously with cultures of the strain of $B$. whitmori cultivated from the patient. An abscess developed at the site of inoculation, in each case, but the local lesion healed and the animals have, so far, remained healthy.

Case 2. The illness commenced gradually with pain after food, occasional vomiting and looseness of the bowels. The temperature became high and of the swinging type, accompanied by rigors and sweats. The liver was enlarged. The patient died on the twenty-fifth day.

At the autopsy, two large abscesses were found in the liver, the gall-bladder was thickened and there were a few small superficial ulcers in the caecum. B. whitmori was cultivated from the liver abscess. No amoebae were found.

The following animals were inoculated. Two wild rats were inoculated subcutaneously with a culture from this case and both died from septicaemia in less than twenty-four hours. B. whitmori was recovered from the heart's blood. The result of the subcutaneous inoculation of a guinea-pig was the same. A guinea-pig, inoculated in the nostril, died on the fifth day with a cheesy deposit in the nose and septicaemia. Another guinea-pig, inoculated in the same way, but after the organism had been subcultivated repeatedly, died on the twelfth day, with caseous tracheal glands containing $B$. whitmori. A rabbit, inoculated subcutaneously, died in twenty-six hours from septicaemia with tubercles in the peritoneum. B. whitmori was cultivated from the peritoneum and from the heart's blood. A monkey fed on a culture remained healthy. A pony inoculated intravenously recovered after a week's fever.

\section{REFERENCES.}

Stanton, A. T. (1917). Studies Inst. Med. Research, Kuala Lumpur, No. 14.

- (1918). Ann. Report Inst. Med. Research, Kuala Lumpur.

and Fletcher, W. (1919-1922). Ann. Reports Inst. Med. Research, Kuala Lumpur. -(1921). Trans. Fourth Congress Far East. Assoc. Trop. Med. II. 196.

Whitmore, A. (1913). Journ. of Hyg. XiII. 1.

— and Krishnaswami, C. S. (1912). Indian Med. Gaz. xuviI. 262.

\section{DESCRIPTION OF PLATES III-V. PLATE III.}

Fig. 1. Bacillus whitmori. Mucoid and corrugated colonies on glycerine agar plate.

PLATE IV.

Figs. 2-5. Illustrating lesions in Case 1.

Fig. 2. Lung, showing areas of consolidation consisting of minute tubercle-like nodules.

Fig. 3. Liver, showing minute suppurating tubercles and thickened gall-bladder.

Fig. 4. Spleen, the white areas are abscesses filled with creamy pus.

Fig. 5. Ileum and ascending colon, with erosions in caecum.

\section{PLATE V.}

Fig. 6. Case 2. Portion of an abscess from the liver showing the thick, necrotic wall.

$$
\text { (MS. received for publication in August 1924.-Ed.) }
$$


JOURNAL OF HYGIENE, VOL. XXIII. NO. 3

PLATE III

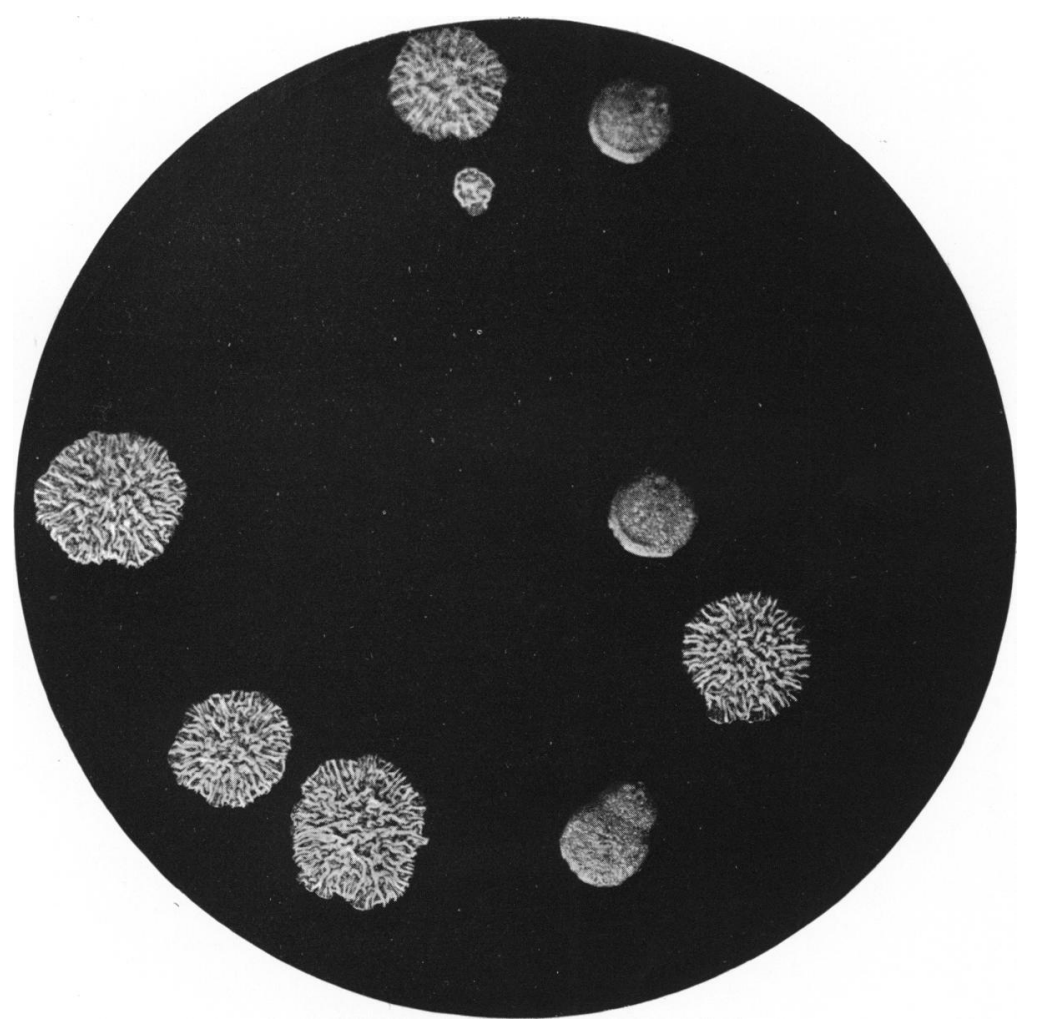

Fig. 1 
JOURNAL OF HYGIENE, VOL. XXIII. NO. 3
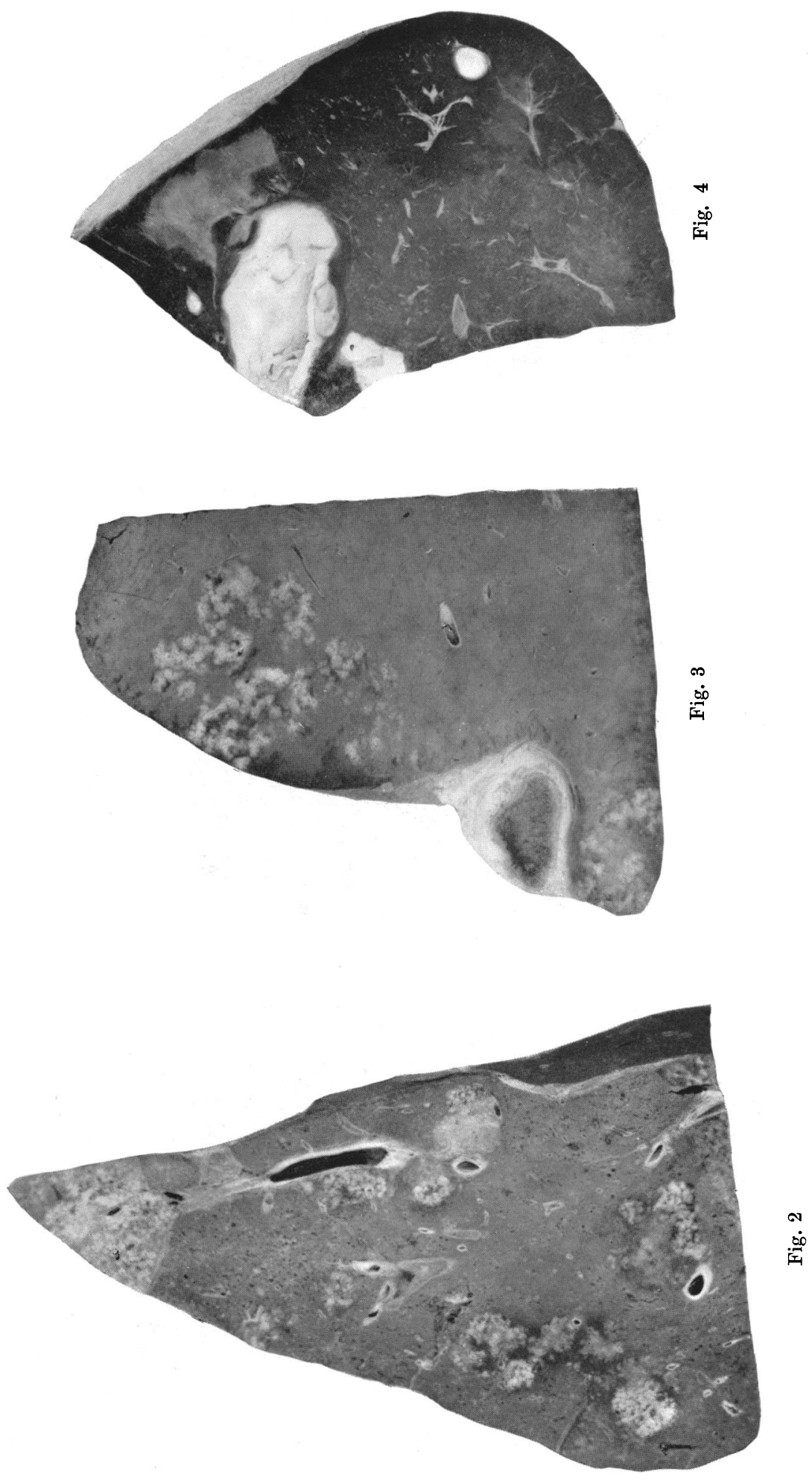


\section{PLATEIV}

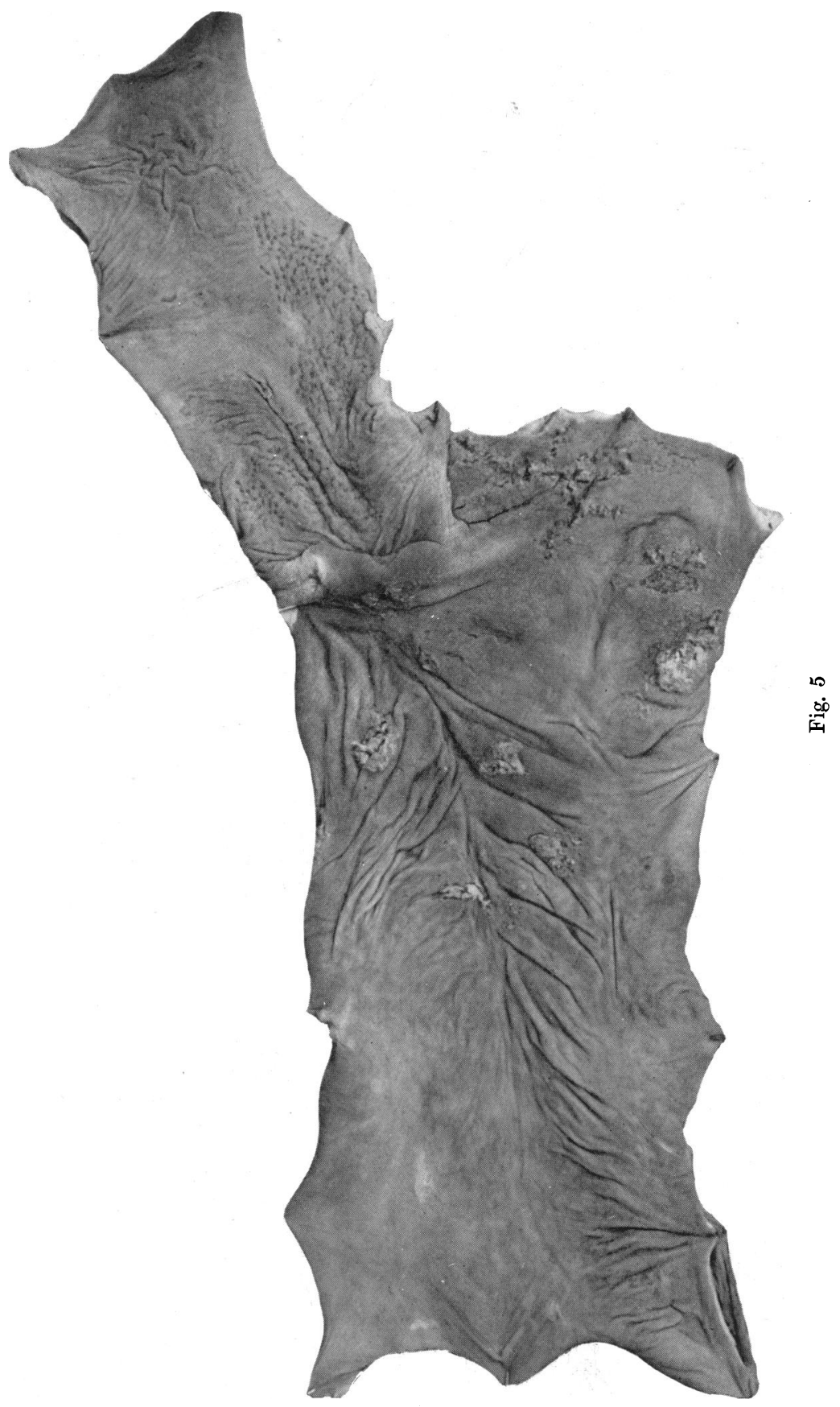


JOURNAL OF HYGIENE, VOL. XXIII. NO. 3

PLATE $V$
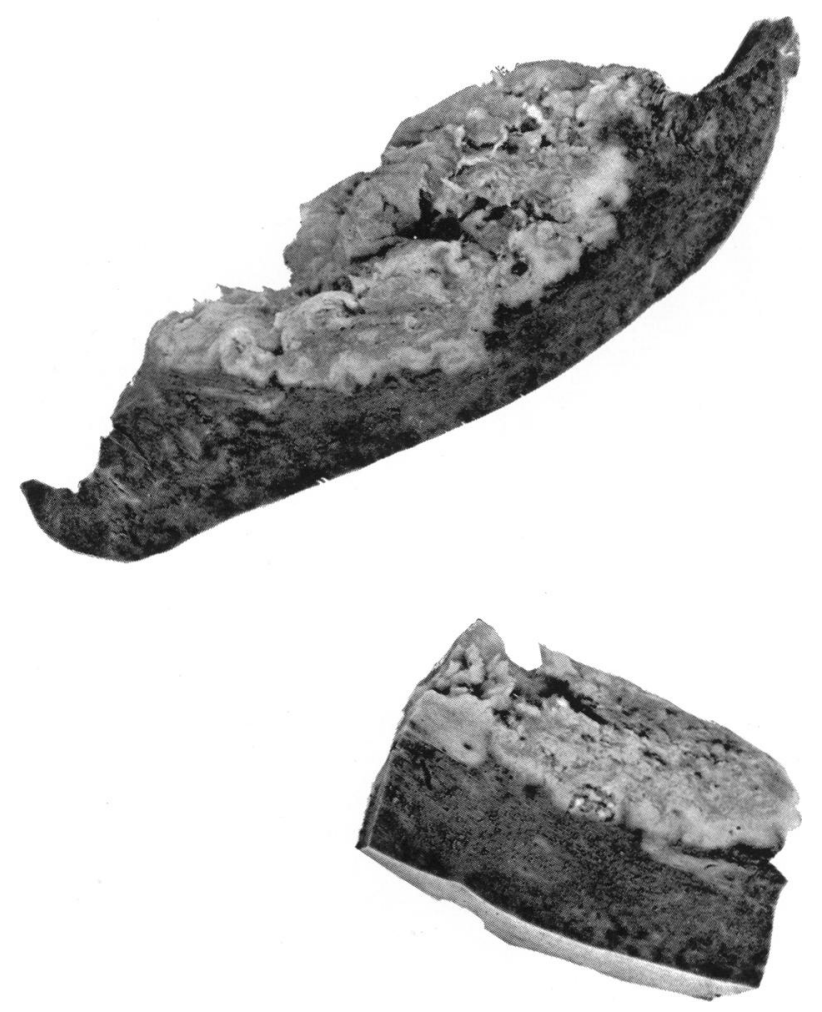

Fig. 6 